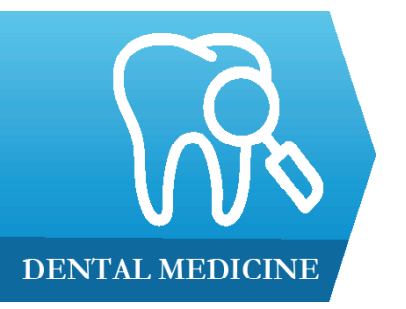

1) Paediatric Dentistry Department, Faculty of Dental Medicine, Iuliu Hatieganu University of Medicine and Pharmacy, Cluj-Napoca, Romania

2) Dental Propaedeutic and Aesthetics Department, Faculty of Dental Medicine, Iuliu Hatieganu University of Medicine and Pharmacy, ClujNapoca, Romania

\section{Pit and fissure sealants penetration capacity and their correlation with fissure morphology}

Alexandrina Muntean ${ }^{1}$, Meda-Romana Simu ${ }^{1}$, Raluca Suhani ${ }^{1}$, Anca Stefania Mesaros ${ }^{2}$

\begin{abstract}
Background and aims. Pit and fissure sealant placement corresponds to an effective approach of the prevention of caries on occlusal surfaces. Fissure morphology and dental material characteristics are the key factors for sealant effectiveness. The aim of this in vitro study was to compare the penetration ability of two commercially available pit and fissure sealants.

Methods. Twenty sound human premolars extracted for orthodontic purpose have been sealed according to manufacturer's instructions as follows: Group I ( $\mathrm{n}=10)$, lightcured unfilled resin-based sealant, Admira Seal ${ }^{\circledR}($ Voco GmbH); Group II $(n=10)$, resin modified glass ionomer sealant without varnish, GC Fuji Triage ${ }^{\circledR}$ (GC Corporation). The teeth have been sectioned buccal-lingually in the middle of the occlusal surface, and the sections were examined at 40x with an inverted microscope.

Results. Penetration of the sealants was found to be greater in U-type fissure pattern (91.69\%) followed by V-type (75.42\%), IK-type (71.24\%) and then in I-type (63.98\%). The depth of penetration of GC Fuji Triage ${ }^{\circledR}(82.85 \%)$ demonstrated to be superior to Admira Seal ${ }^{\circledR}(76.28 \%)$.

Conclusion. $U$ fissure design was more common than other fissure patterns and showed significantly higher penetration for the two type of sealants evaluated. Resin modified glass ionomer sealant used in the present study perform comparably with the filled resin sealant.
\end{abstract}

Keywords: dental sealant, fissure patterns, penetration

\section{Background and aims}

Dental caries is a multifactorial disease caused by the alteration in the composition of the bacterial biofilm, leading to an imbalance between the demineralization and remineralization processes. The phenomenon is manifested by the formation of carious lesions in primary and permanent dentitions [1]. Pit and fissure caries correspond to about $90 \%$ of the caries of permanent posterior teeth and $44 \%$ of caries in the primary teeth in children and adolescents [2]. The use of caries preventive measures, such as topical fluoride therapy, plaque control, and dietary sugar control, are generally considered the cause of the overall decline of caries prevalence. The plaque retentive nature of pits and fissures render cleaning difficult, causing them to be more susceptible to caries than smooth surfaces and most likely not protected by fluoride administration [1,2].

Dental sealants appear to be a costeffective intervention; sealing permanent molars reduces the total cost by preventing the need for more expensive and invasive restorative treatment. The use of sealants should be selective, based on the child's caries risk and fissures anatomy [3].

Perception of the susceptibility of pits and fissures to caries varies from practitioner to practitioner, when simple terms such as "deep occlusal anatomy" are used. In every day practice, we should be aware of the teeth surfaces that are most susceptible to caries and include them in treatment plan, with specific prophylactic methods. Sealant application is part of caries management protocol, in accordance with patient caries risk $[2,4]$.

A study showed that sealing 
permanent molars in all patients further improves the outcome, adding only a small incremental cost relative to risk-based sealing [5]. Another study concluded that sealing primary molars reduces restorations and extractions and ensures an oral environment with less risk factors for recently erupted permanent teeth [6].

Fissure morphology is an element intrinsically related to decay susceptibility and progression. Deep, narrow, IKshaped fissures or I, are relatively more caries-susceptible, compared to, wide, U-shaped fissures [7]. Newly erupted permanent first molars should be seen as susceptible teeth, prior to full eruption and must be sealed at an early or late stage of this natural process [6,7]. The aim of this in vitro study was to evaluate sealant penetration in accordance with fissure anatomy, in order to encourage sealant application even in clinical situation judged as inappropriate.

\section{Methods}

Patient informed consent for orthodontic extraction were obtained prior to treatment initiation. Twenty freshly human first maxillary premolars extracted for orthodontic purpose were prepared after a specific protocol and included in the study[8]. The teeth were clinically evaluated under standard lighting condition with an explorer and were found caries free. The teeth had been cleaned by removing calculus and soft tissue deposits with a hand scaler, and then stored in $0.9 \% \mathrm{NaCl}$ containing $0.02 \%$ sodium azide at $4^{\circ} \mathrm{C}$ until used. Teeth were prepared for prophylaxis using pumice and Klint Paste ${ }^{\circledR}$ (Voco) for surface cleaning. Dental sealants were applied on pit and fissures following the manufacturers' recommendations:

Group I: the occlusal grooves were etched with $37 \%$ phosphoric acid gel for $30 \mathrm{~s}$, rinsed with water spray for 20 $\mathrm{s}$ and dried with a gentle air stream for $10 \mathrm{~s}$. One layer of light-cured unfilled resin-based sealant (RBS) Admira Seal ${ }^{\circledR}$ (Voco) was applied using a special applicator with a light brushing motion, and then light cured for $20 \mathrm{~s}$ using Optilux 501curing unit (Kerr Corp.).

Group II: the occlusal grooves were conditioned with GC Cavity Conditioner (20\% polyacrylic acid and 3\% aluminum chloride hexahydrate) for $10 \mathrm{~s}$, then washed for 20 $\mathrm{s}$ and dried but not desiccated with a gentle air stream for 3 $\mathrm{s}$, in order to obtain a moist surface. The resin modified glass ionomer sealant (RMGI) GC Fuji Triage ${ }^{\circledR}$ was prepared according to the manufacturer's recommendations and one layer was applied with an explorer, and then light cured for 20 s using Optilux 501 curing unit (Kerr Corp) [8].

The light-curing unit, Optilux 501(Kerr Corp), was operated in the standard mode at a light intensity of $740 \pm$ $36 \mathrm{~mW} / \mathrm{cm} 2$. The light curing unit output was measured after every five procedures using a Kerr LED hand-held radiometer (Kerr Corp) as specified by the producer.

The teeth were thermocycled between $50^{\circ} \mathrm{C}$ and $55^{\circ} \mathrm{C}$ for 1000 cycles with a dwell time of $25 \mathrm{~s}$. The apices of the teeth were sealed with resin composite and the tooth surfaces were covered with two layers of nail varnish with the exception of $1 \mathrm{~mm}$ around the tooth-sealant interface. The teeth were immersed in $0.5 \%$ basic fuchsine dye for $24 \mathrm{~h}$. They were removed, washed, dried and their roots mounted in self-curing acrylic resin. Each premolar was sectioned buccal-lingually using a water-cooled microtome (Isomet Low Speed Saw, Buehler Ltd) in order to obtain a $1.5 \mathrm{~mm}$ thick section in the middle of the occlusal surface. A single examiner assessed each section using an inverted microscope (Olympus KC301, Olympus America Inc.) at 40X [8]. Several linear parameters were measured and recorded in microns $(\mu)$ using a QuickPhoto Micro 2.2 software (Olympus Inc) as follows:

- Sealant penetration depth $(\mu)$ calculated as length measured $(\mu)$ from the deepest point on concavity of the upper margin of the occlusal sealant to the base of the sealant

- Length of Unfilled space $(\mu)$ calculated as length measured $(\mu)$ from base of the sealant to the base of the fissure

- Total length of fissure $(\mu)$ calculated as length measured $(\mu)$ from deepest point on the upper margin of the sealant to the base of the fissure

- Penetrability $(\%)=($ Sealant Penetration Depth $/$ Total Length of Fissure) X 100 [9].

Three measurements of each section were performed and the mean value per section was assumed as the representative values. Data were collected and the statistical analysis was carried out by using Statistical Packages for Social Sciences (SPSS 13.0, Chicago, IL, USA) for Windows. Average values for each group were subjected to statistical analysis by Student's t and Kolmogorov-Smirnov test at a $\mathrm{p}<0.05$ level of significance.

\section{Results}

First microscopic observation revealed various shapes and morphologies for occlusal first maxillary premolar surfaces (Figure 1, Figure 2, Table I).

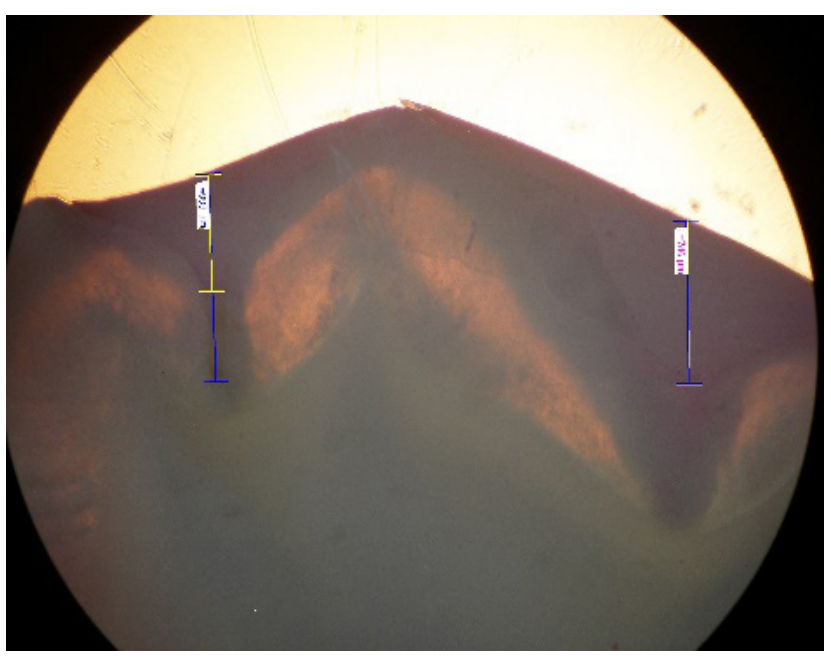

Figure 1. Fissure morphology and penetration for RBS sealant. 


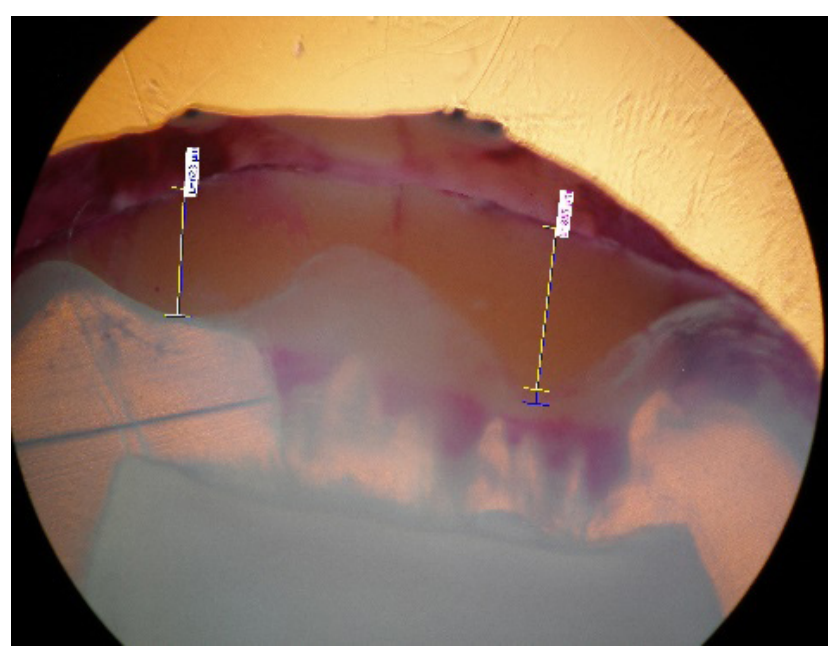

Figure 2. Fissure morphology and penetration for RMGI sealant.

Table I. Fissure patterns in study group.

\begin{tabular}{l|c} 
Fissure type & Proportion in the study group \\
\hline U-type & $37.3 \%$ \\
V-type & $29.9 \%$ \\
I-type & $23.9 \%$ \\
IK-type & $8.9 \%$
\end{tabular}

Differences regarding sealant penetrability appear to be in direct correlation with fissure shape. We observe that no material was able to penetrate down to the bottom, especially in deep and narrow fissures (Table II).

Table II. Sealant penetrability correlated with fissure pattern.

\begin{tabular}{l|c|} 
Fissure type & Penetrability \\
\hline U-type & $91.69 \%$ \\
V-type & $75.42 \%$ \\
IK-type & $71.24 \%$ \\
I-type & $63.98 \%$
\end{tabular}

Regarding the penetrability of the dental sealants, we notice that RMGIC perform comparably with RBS, with no significant statistical differences (Table III).

\section{Discussion}

Caries remains a prevalent disease, affecting every geographic region, regardless of age and socioeconomic status. Untreated caries in the permanent dentition was the most prevalent disease in the world in the past decade, while untreated caries in the deciduous dentition was classified the tenth most prevalent[2,4]. Despite advancements in preventive methods and dental material technologies, caries prevalence and incidence have remained high in many countries, and Romania is no exception to the rule $[2,10]$. Caries is regarded as a 'non-communicable' and 'behavioral' disease of the oral cavity. Decay initiation involves net demineralization of the dental hard tissue, which occurs because of $\mathrm{pH}$ imbalance or acidity-facilitated cariogenic bacteria in the presence of fermentable sugars $[3,10]$.

"Prevention is better than cure"- the medical model of caries management addresses the preventive aspect of the caries disease. Healthy dental tissues protection against caries depend on fluoride application, fissure sealants and dietary alterations or improvements. The surgical model claims to be of a preventive nature by cutting away "caries-prone fissures", this does not avoid new caries from initiating. On the contrary, the surgical model weakens the tooth structure by cutting natural tooth substance $[2,10,11]$.

Occlusal surfaces comprise only $12 \%$ of the total tooth surface in the mouth; however, they are eight times more vulnerable to caries than the smooth surfaces. Fissure sealants proved to be effective in the prevention of pit and fissure caries. Systematic reviews have shown that dental sealants are effective in reducing caries incidence with similar outcomes between resin and glass ionomer varieties $[2,10,11]$. Glass ionomer sealants might possibly have an additional cariostatic benefit by local fluoride release. Sealants reduce caries risk by mechanically blocking the accumulation and formation of cariogenic plaque from uncleanable deep pits and fissures and alter the micro environment within the fissure into a cariostatic one $[2,11,12]$.

The occlusal fissure pattern has been classified in literature based on fissure morphology as follows: $\mathrm{V}, \mathrm{U}$, I (Y1), IK (Y2) and inverted Y [7,9]. For teeth evaluated in our study, fissure shape U (37.3\%), V (29.9\%) and IK $(23.9 \%)$ were encountered most frequently, elements that encourage sealant application.

Table III. Correlation between fissure pattern and dental sealant features.

\begin{tabular}{l|c|c|c|c} 
& U-type & V-type & IK-type & I-type \\
\hline Admira Seal & $90.45 \pm 7.13$ & $68.58 \pm \mathbf{2 8 . 0 8}$ & $64.52 \pm 9.11$ & $58.25 \pm \mathbf{0 . 5 9}$ \\
Fuji & $92.89 \pm 3.69$ & $82.26 \pm 12.64$ & $77.96 \pm 23.96$ & $69.71 \pm \mathbf{1 3 . 3 4}$ \\
p & 0.07 & 0.39 & 0.34 & 0.19
\end{tabular}


Complete penetration of the sealant into complex fissure systems, especially deep and narrow fissures, is difficult compared to wide and shallow fissures, due to the phenomenon of closed end capillaries or isolated capillaries. Our results show values higher than $70 \%$ penetration for $\mathrm{U}, \mathrm{V}$ and IK fissures, but we have to consider dental material characteristics (surface tension and viscosity) especially in I and IK fissure patterns, which can be correlated with other research paper $[13,14]$. This in vitro study also proves that occlusal morphology is a limiting factor for penetration of fissure sealants. Maximum depth of penetration, good adaptation and limited marginal microleakage are more important than the complete penetration of the sealant to the base of the fissure $[15,16]$.

Resin-based materials have high retention and superior wear resistance, but are clinically limited by the difficulties in their use, due to the technique sensitivity, as these materials are primarily hydrophobic and require a dry field [16]. Although resin modified glass ionomer pit and fissure sealant has the advantage of antibacterial property, better handling, free flowing consistency and improved adherence to enamel $[17,18]$, resin modified glass ionomer sealant performed better under wet contamination conditions compared to resin-based sealant. Consequently, this dental material type could be a better choice while treating pediatric patients $[16,19]$.

Our results are in line with literature data; penetrability for the GC Fuji VII ${ }^{\circledR}$ glass ionomer sealant was slightly higher, but without significant statistical differences, when compared with resin-based sealant Admira Seal ${ }^{\circledR}[9,19]$. The results in our study advocate that the overall penetration of GC Fuji VII ${ }^{\circledR}$ sealants is more than $70 \%$ of entire fissure depth in $\mathrm{U}, \mathrm{V}$ and IK fissures, which can be correlated with other research papers and encourage the use of this prophylactic method, even for morphological occlusal details considered as inadequate $[9,20]$.

Penetration depth is an important parameter that may increase the longevity, the retention and the adaptation of the sealant of sealant. The objective evaluation of penetrability allows a better characterization of dental materials used as dental sealants. In our study the fissure did not undergo mechanical preparation, and for this reason, the results were more than encouraging. We can assume that in vitro condition and sealant application by a single operator represented factors that contribute to higher penetration values. Resin modified glass ionomer sealant could be used as a transitional sealant in specific conditions, especially in high caries risk uncooperative patients, even if mechanical properties of this material did not reach the accomplishment of resin based sealant $[19,20]$.

\section{Conclusions}

Pit and fissure sealant placement represented an effective approach in preventive dentistry. Material physical characteristics and fissure morphology were significant factors as regards sealant penetrability. $U$ and V-type fissures display the most favorable conditions for sealant application, as they are shallow. Despite the limitation of this in vitro study, sealants used in pediatric patients must be encouraged, in order to control caries risk and reduce treatment needs for recently erupted permanent teeth.

\section{References}

1. Muntean A, Mesaros AS, Festila D, Mesaros M. Modern management of dental decay in children and adolescents - a review. Clujul Med. 2015;88:137-139.

2. Yon MJY, Gao SS, Chen KJ, Duangthip D, Lo ECM, Chu $\mathrm{CH}$. Medical Model in Caries Management. Dent J (Basel). 2019;7. doi: 10.3390/dj7020037.

3. Cvikl B, Moritz A, Bekes K. Pit and Fissure Sealants - A Comprehensive Review. Dent J (Basel). 2018;6. doi: 10.3390/dj6020018.

4. American Academy of Pediatric Dentistry. Guideline on caries-risk assessment and management for infants, children, and adolescents. Pediatr Dent. 2014;36:127-134.

5. Chestnutt IG, Playle R, Hutchings S, Morgan-Trimmer S, Fitzsimmons D, Aawar N, et al. Fissure Seal or Fluoride Varnish? A Randomized Trial of Relative Effectiveness. J Dent Res. 2017;96:754-761.

6. Chi DL, van der Goes DN, Ney JP. Cost-effectiveness of pitand-fissure sealants on primary molars in Medicaid-enrolled children. Am J Public Health. 2014;104:555-561.

7. Nagano T. Relation between the Form of Pit and Fissure and the Primary Lesion of Caries. Dent Abstr.1961;6:4265.

8. Muntean A, Mesaros A, Festila D, Moldovan M, Mesaros M. In vitro microleakage evaluation, around three types of dental sealants. Mat Plastice. 2016;1:166-169.

9. Garg N, Indushekar KR, Saraf BG, Sheoran N, Sardana D. Comparative Evaluation of Penetration Ability of Three Pit and Fissure Sealants and Their Relationship with Fissure Patterns. J Dent (Shiraz). 2018;19:92-99.

10. Kassebaum NJ, Bernabé E, Dahiya M, Bhandari B, Murray CJ, Marcenes W. Global burden of untreated caries: a systematic review and metaregression. J Dent Res. 2015;94:650-658.

11. Ahovuo-Saloranta A, Forss H, Walsh T, Nordblad A, Mäkelä M, Worthington HV. Pit and fissure sealants for preventing dental decay in permanent teeth. Cochrane Database Syst Rev. 2017;7:CD001830.

12. Wright JT, Crall JJ, Fontana M, Gillette EJ, Nový BB, Dhar $\mathrm{V}$, et al. Evidence-based clinical practice guideline for the use of pit-and-fissure sealants: A report of the American Dental Association and the American Academy of Pediatric Dentistry. J Am Dent Assoc. 2016;147:672-682.

13. Pardi V, Sinhoreti MA, Pereira AC, Ambrosano GM, 
Meneghim Mde C. In vitro evaluation of microleakage of different materials used as pit-and-fissure sealants. Braz Dent J. 2006; 17:49-52.

14. Zhao L, Shi Q. Microleakage and penetration ability of different sealants. Int J Ped Dent. 2009;19:71-75.

15. Grewal N, Chopra R. The effect of fissure morphology and eruption time on penetration and adaptation of pit and fissure sealants: An SEM study. J Indian Soc Pedod Prev Dent. 2008;26:59-63.

16. da Silveira Ana Daniela Silva, Fagundes Nathália Carolina Fernandes, Lascala Cesar Angelo, da Silva Nogueira Antônio José, Marques Marcia Martins, de Cerqueira Luz Maria Aparecida Alves. Effect of a resin-based and a glassionomer sealant on the treatment of noncavitated occlusal caries lesions in teenagers. 2017;6:48-53.
17. Godhane A, Ukey A, Tote JV, Das G, Naphde M, Patil P. Use of Pit and Fissure Sealant in Prevention of Dental Caries in Pediatric Dentistry and Recent Advancement: A Review. Int J Dent Med Res. 2015;1:220-223.

18. Baygin O, Korkmaz FM, Tüzüner T, Tanriver M. The effect of different enamel surface treatments on the microleakage of fissure sealants. Lasers Med Sci. 2012;27:153-160.

19. Al-Jobair A, Al-Hammad N, Alsadhan S, Salama F. Retention and caries-preventive effect of glass ionomer and resin-based sealants: An 18-month-randomized clinical trial. Dent Mater J. 2017;36:654-661.

20. Markovic D, Petrovic B, Peric T, Miletic I, Andjelkovic $\mathrm{S}$. The impact of fissure depth and enamel conditioning protocols on glass-ionomer and resin-based fissure sealant penetration. J Adhes Dent. 2011;13:171-178. 